\title{
$V$-Fuzzy metric space and related fixed point theorems
}

\author{
Vishal Gupta* and Ashima Kanwar
}

"Correspondence:

vishal.gmn@gmail.com

Department of Mathematics,

Maharishi Markandeshwar

University, Mullana, Ambala,

Haryana 133207, India

\begin{abstract}
The paper concerns our sustained efforts for introduction of $V$-fuzzy metric spaces and to study their basic topological properties. As an application of this concept, we prove coupled common fixed point theorems for mixed weakly monotone maps in partially ordered $V$-fuzzy metric spaces. An example quoted in this paper also corroborates fully the main result. Also, here we introduce the concept of a symmetric $V$-fuzzy metric space. Under the influence of symmetry property of $V$-fuzzy metric spaces, its conversion materializes to the main result from $V$-fuzzy metric spaces to fuzzy metric spaces.
\end{abstract}

MSC: Primary $47 \mathrm{H} 10$; secondary $54 \mathrm{H} 25$

Keywords: partial ordered set; mixed monotone mappings; common coupled fixed point; G-fuzzy metric space; $V$-fuzzy metric space

\section{Introduction and preliminaries}

A metric space is just a nonempty set $X$ associated with a function $d$ of two variables enabling us to measure the distance between points. In advanced mathematics, we need to find the distance not only between numbers and vectors, but also between more complicated objects like sequences, sets, and functions. In order to find an appropriate concept of a metric space, numerous approaches exist in this sphere. Thus, new notions of distance lead to new notions of convergence and continuity. A number of generalizations of a metric space have been discussed by many eminent mathematicians. Mustafa and Sims [1] introduced the notion of a G-metric space and suggested an important generalization of a metric space as follows.

Definition 1.1 [1] Let $X$ be a nonempty set, and let $G: X^{3} \rightarrow[0,+\infty)$ be a function satisfying the following conditions for all $x, y, z, a \in X$ :

(G-1) $G(x, y, z)=0$ if $x=y=z$,

(G-2) $0 \leq G(x, y, z)$ with $x \neq y$,

(G-3) $G(x, x, y) \leq G(x, y, z)$ with $y \neq z$,

(G-4) $G(x, y, z)=G(x, z, y)=G(y, x, z)=G(z, x, y)=G(y, z, x)=G(z, y, x)$,

(G-5) $G(x, y, z) \leq G(x, a, a)+G(a, y, z)$.

The function $G$ is called a generalized metric on $X$, and the pair $(X, G)$ is called a G-metric space. (c) 2016 Gupta and Kanwar. This article is distributed under the terms of the Creative Commons Attribution 4.0 International License (http://creativecommons.org/licenses/by/4.0/), which permits unrestricted use, distribution, and reproduction in any medium, provided you give appropriate credit to the original author(s) and the source, provide a link to the Creative Commons license, and indicate if changes were made. 
Example 1.1 [1] Let $(X, d)$ be a metric space. Define $G: X \times X \times X \rightarrow R^{+}$by

$$
G(x, y, z)=\frac{d(x, y)+d(y, z)+d(z, x)}{3} .
$$

Then $(X, G)$ is a $G$-metric space.

Recently, some authors studied some important fixed point theorems with application in G-metric spaces [2-4]. In 2012, Sedghi et al. [5] introduced a new generalized metric space called an $S$-metric space.

Definition 1.2 [5] Let $X$ be a nonempty set. Suppose that a function $S: X^{3} \rightarrow[0,+\infty)$ satisfies the following conditions:

$(\mathrm{S}-1) \quad S(x, y, z) \geq 0$,

(S-2) $S(x, y, z)=0$ if and only if $x=y=z=0$,

(S-3) $S(x, y, z) \leq S(x, x, a)+S(y, y, a)+S(z, z, a)$ for any $x, y, z, a \in X$.

Then the ordered pair $(X, S)$ is called an $S$-metric space.

Example 1.2 [5] Let $\Re$ be the real line. Then $S(x, y, z)=|x-y|+|y-z|$ for all $x, y, z \in \Re$ is an $S$-metric on $\Re$. This $S$-metric is called the usual $S$-metric on $\Re$.

Abbas et al. [6] established the notion of $A$-metric spaces, a generalization of $S$-metric spaces.

Definition 1.3 [6] Let $X$ be a nonempty set. A function $A: X^{n} \rightarrow[0,+\infty)$ is called an $A$-metric on $X$ if for any $x_{i}, a \in X, i=1,2,3, \ldots, n$, the following conditions hold:

(A-1) $A\left(x_{1}, x_{2}, x_{3}, \ldots, x_{n}\right) \geq 0$,

(A-2) $A\left(x_{1}, x_{2}, x_{3}, \ldots, x_{n}\right)=0$ if and only if $x_{1}=x_{2}=x_{3}=\cdots=x_{n}=0$,

(A-3) $A\left(x_{1}, x_{2}, x_{3}, \ldots, x_{n}\right) \leq A\left(x_{1}, x_{1}, x_{1}, \ldots,\left(x_{1}\right)_{n-1}, a\right)+A\left(x_{2}, x_{2}, x_{2}, \ldots,\left(x_{2}\right)_{n-1}, a\right)+\cdots+$ $A\left(x_{n}, x_{n}, x_{n}, \ldots,\left(x_{n}\right)_{n-1}, a\right)$.

The pair $(X, A)$ is called an $A$-metric space.

Example 1.3 [6] Let $X=\Re$. Define the function $A: X^{n} \rightarrow[0,+\infty)$ by

$$
A\left(x_{1}, x_{2}, x_{3}, \ldots, x_{n}\right)=\sum_{i=1}^{n} \sum_{i<j}\left|x_{i}-y_{j}\right|
$$

Then $(X, A)$ is called the usual $A$-metric space.

Fixed point theorems have been studied in many contexts, one of which is the fuzzy setting. The concept of fuzzy sets was initially introduced by Zadeh [7] in 1965. To use this concept in topology and analysis, the theory of fuzzy sets and its applications have been developed by many eminent authors. It is well known that a fuzzy metric space is an important generalization of a metric space.

Many authors have introduced fuzzy metric spaces in different ways. For instance, George and Veeramani [8] modified the concept of a fuzzy metric space introduced by Kramosil and Michalek [9] and defined the Hausdorff topology of a fuzzy metric space. 
Definition 1.4 [10] A binary operation $*:[0,1] \times[0,1] \rightarrow[0,1]$ is called a continuous $t$-norm if $*$ satisfies following conditions:

(i) $*$ is commutative and associative,

(ii) $*$ is continuous,

(iii) $a * 1=a, \forall a \in[0,1]$,

(iv) $a * b \leq c * d$ whenever $a \leq c$ and $b \leq d$ for all $a, b, c, d \in[0,1]$.

Definition 1.5 [9] The triplet $(X, M, *)$ is called a fuzzy metric space (shortly, FM-space) if $X$ is an arbitrary set, $*$ is a continuous $t$-norm, and $M$ is a fuzzy set in $X \times X \times[0, \infty)$ satisfying the following conditions for all $x, y, z \in X$ and $s, t>0$ :

$(\mathrm{FM}-1) \quad M(x, y, 0)=0$,

(FM-2) $M(x, y, t)=1$ if and only if $x=y$,

(FM-3) $M(x, y, t)=M(y, x, t)$,

$(\mathrm{FM}-4) \quad M(x, y, t) * M(y, z, s) \leq M(x, z, t+s)$,

$(\mathrm{FM}-5) M(x, y, \cdot):[0, \infty) \rightarrow[0,1]$ is left continuous.

Note that $M(x, y, t)$ can be thought of as a degree of nearness between $x$ and $y$ with respect to $t$.

Example 1.4 [8] Let $(X, d)$ be a metric space. Define the $t$-norm $a * b=a b$ or $a * b=$ $\min \{a, b\}$. For all $x, y \in X, t>0$, let

$$
M(x, y, t)=\frac{t}{t+d(x, y)} .
$$

Then $(X, M, *)$ is a fuzzy metric space.

Lemma 1.1 [11] Let $(X, M, *)$ be a fuzzy metric space. If there exists $k \in(0,1)$ such that, for all $x, y \in X$ and $t>0$,

$$
M(x, y, k t) \geq M(x, y, t)
$$

for all $x, y \in X, t>0$, then $x=y$.

In the process of generalization of fuzzy metric spaces, Sun and Yang [12] coined the notion of G-fuzzy metric spaces and established common fixed-point theorems for four mappings.

Definition 1.6 [12] A triplet $(X, V, *)$ is said to be a $G$-fuzzy metric space (shortly, GF-space) if $X$ is an arbitrary nonempty set, $*$ is a continuous $t$-norm, and $G$ is a fuzzy set on $X \times X \times X \times[0, \infty)$ satisfying the following conditions for all $x, y, z \in X$ and $s, t>0$ :

(GF-1) $G(x, x, y, t)>0$ with $x \neq y$,

(GF-2) $G(x, x, y, t) \geq G(x, y, z, t)$ with $y \neq z$,

(GF-3) $G(x, y, z, t)=1$ if and only if $x=y=z$,

(GF-4) $G(x, y, z, t)=G(x, z, y, t)=G(y, x, z, t)=G(z, x, y, t)=G(y, z, x, t)=G(z, y, x, t)$,

(GF-5) $G(x, y, z, t+s) \geq G(x, a, a, t) * G(a, y, z, s)$,

$(\mathrm{GF}-6) \mathrm{G}(x, y, z, \cdot):[0, \infty) \rightarrow[0,1]$ is left continuous. 
Example 1.5 [12] Let $G$ be a $G$-metric on a nonempty set $X$. Define the $t$-norm $a * b=$ $\min \{a, b\}$. For all $x, y \in X$ and $t>0$, denote

$$
G(x, y, z, t)=\frac{t}{t+G(x, y, z)} .
$$

Then $(X, G, *)$ is a $G F$-space.

Lemma 1.2 [12] Let $(X, G, *)$ be a GF-space. Then $G(x, y, z, t)$ is nondecreasing with respect to $t$ for all $x, y, z \in X$.

On the other hand, the concepts of coupled fixed points and mixed monotone property of a fuzzy metric space are established by Bhaskar and Lakshmikantham [13]. Lakshmikantham and Ciric [14] discussed the mixed monotone mappings and gave some coupled fixed point theorems, which can be used to discuss the existence and uniqueness of a solution for a periodic boundary value problem.

Definition 1.7 [14] An element $(x, y) \in X \times X$ is called a coupled fixed point of a mapping $P: X \times X \rightarrow X$ if $P(x, y)=x$ and $P(y, x)=y$.

Definition 1.8 [14] An element $(x, y) \in X \times X$ is called a coupled coincidence point of the mappings $P: X \times X \rightarrow X$ and $Q: X \rightarrow X$ if $P(x, y)=Q(x)$ and $P(y, x)=Q(y)$.

Definition 1.9 [14] An element $(x, y) \in X \times X$ is called a common coupled fixed point of the mappings $P: X \times X \rightarrow X$ and $Q: X \rightarrow X$ if $x=P(x, y)=Q(x)$ and $y=P(y, x)=Q(y)$.

Example 1.6 Let $X=[0,1]$. Define $P: X \times X \rightarrow X$ and $Q: X \rightarrow X$ as

$$
P(x, y)=\left|\frac{x-y}{2}+\frac{1}{4}\right|, \quad Q(x)=x .
$$

For $x=0$ and $y=\frac{1}{2}$, we have

$$
P(x, y)=\left|\frac{0-\frac{1}{2}}{2}+\frac{1}{4}\right|=0, \quad Q(0)=0
$$

and

$$
P(y, x)=\left|\frac{\frac{1}{2}-0}{2}+\frac{1}{4}\right|=\frac{1}{2}, \quad Q\left(\frac{1}{2}\right)=\frac{1}{2} .
$$

So, $(x, y)=\left(0, \frac{1}{2}\right)$ is a common coupled fixed point of the mappings $P: X \times X \rightarrow X$ and $Q: X \rightarrow X$.

Definition 1.10 [14] An element $x \in X$ is called a common fixed point of the mappings $P: X \times X \rightarrow X$ and $Q: X \rightarrow X$ if $x=P(x, x)=Q(x)$.

Example 1.7 Define $P: X \times X \rightarrow X$ and $Q: X \rightarrow X$ where $X=[0,1]$ as

$$
P(x, y)=x-\frac{y}{2}+\frac{x y}{2}, \quad Q(x)=x .
$$


Then we have

$$
P(x, x)=\frac{x+x^{2}}{2}, \quad Q(x)=x
$$

For $x=1$, we get $P(1,1)=1=Q(1)$. So, $(x, y)=(1,1)$ is a common fixed point of the pair $(P, Q)$.

Definition 1.11 [13] Let $(X, \preceq)$ be a partially ordered set. The mapping $(x, y)$ is said to have the mixed monotone property if $P$ is monotone nondecreasing in its first argument and is monotone nonincreasing in its second argument; that is, for any $x, y \in X$,

$$
x_{1}, x_{2} \in X, \quad x_{1} \preceq x_{2} \quad \Longrightarrow \quad P\left(x_{1}, y\right) \preceq P\left(x_{2}, y\right)
$$

and

$$
y_{1}, y_{2} \in X, \quad y_{1} \preceq y_{2} \quad \Longrightarrow \quad P\left(x, y_{1}\right) \succeq P\left(x, y_{2}\right) .
$$

Example 1.8 Let $P: X \times X \rightarrow X$ where $X=[0,1]$ be defined by

$$
P(x, y)= \begin{cases}x-y & \text { if } x \geq y, \\ 0 & \text { if } x \prec y .\end{cases}
$$

For $x_{1}, x_{2} \in X, x_{1} \preceq x_{2}$, we have

$$
x_{1}-y \preceq x_{2}-y \quad \Longrightarrow \quad P\left(x_{1}, y\right) \preceq P\left(x_{2}, y\right) \quad \text { and } \quad P\left(x, y_{1}\right) \succeq P\left(x, y_{2}\right) \text {. }
$$

Therefore, the mapping $P$ has the mixed monotone property.

Definition 1.12 [13] Let $(X, \preceq)$ be a partially ordered set, and $P: X \times X \rightarrow X$ and $Q: X \rightarrow X$. We say that $P$ has the mixed $Q$-monotone property if $P$ is monotone $Q$-nondecreasing in its first argument and is monotone $Q$-nonincreasing in its second argument, that is, for any $x, y \in X$,

$$
x_{1}, x_{2} \in X, \quad Q\left(x_{1}\right) \preceq Q\left(x_{2}\right) \quad \Longrightarrow \quad P\left(x_{1}, y\right) \preceq P\left(x_{2}, y\right)
$$

and

$$
y_{1}, y_{2} \in X, \quad Q\left(y_{1}\right) \preceq Q\left(y_{2}\right) \quad \Longrightarrow \quad P\left(x, y_{1}\right) \succeq P\left(x, y_{2}\right) .
$$

Example 1.9 Let $P: X \times X \rightarrow X$ and $Q: X \rightarrow X$ where $X=[-1,1]$ be two functions given by

$$
P(x, y)=x-y^{2}, \quad Q(x)=x^{4} .
$$

Therefore, the map $P$ has the mixed $Q$-monotone property. 
Definition 1.13 [15] The mappings $P: X \times X \rightarrow X$ and $Q: X \rightarrow X$ are said to be $W$-compatible if

$$
Q(P(x, y))=P(Q x, Q y)
$$

and

$$
Q(P(y, x))=P(Q y, Q x)
$$

whenever $P(x, y)=Q(x)$ and $P(y, x)=Q(y)$ for some $(x, y) \in X \times X$.

Example 1.10 Define $P: X \times X \rightarrow X$ and $Q: X \rightarrow X$ where $X=[-1,1]$ as $P(x, y)=\frac{x^{2}+y^{2}}{2}$, $Q(x)=x$, which satisfies $Q(P(x, y))=P((Q x, Q y))$ and $Q(P(y, x))=P((Q y, Q x))$. For $x=1$ and $y=1$, we get $P(x, y)=Q(x)$ and $P(y, x)=Q(y)$. This implies that the mappings $P: X \times X \rightarrow X$ and $Q: X \rightarrow X$ are $W$-compatible mappings.

Many others eminent authors proved significant results which contributed in the arena of fixed point theory (see [16-21]).

\section{V-Fuzzy metric space}

After the exhaustive review of the previous literature, we introduce $V$-fuzzy metric spaces and discuss their properties given below.

Definition 2.1 Let $X$ be a nonempty set. $A$ triple $(X, V, *)$ is said to be a $V$-fuzzy metric space (denoted by VF-space), where $*$ is a continuous $t$-norm, and $V$ is a fuzzy set on $X^{n} \times(0, \infty)$ satisfying the following conditions for all $t, s>0$ :

(VF-1) $V(x, x, x, \ldots, x, y, t)>0$ for all $x, y \in X$ with $x \neq y$;

(VF-2) $V\left(x_{1}, x_{1}, x_{1}, \ldots, x_{1}, x_{2}, t\right) \geq V\left(x_{1}, x_{2}, x_{3}, \ldots, x_{n}, t\right)$ for all $x_{1}, x_{2}, x_{3}, \ldots, x_{n} \in X$ with $x_{2} \neq x_{3} \neq \cdots \neq x_{n}$

(VF-3) $V\left(x_{1}, x_{2}, x_{3}, \ldots, x_{n}, t\right)=1$ if and only if $x_{1}=x_{2}=x_{3}=\cdots=x_{n}$;

(VF-4) $V\left(x_{1}, x_{2}, x_{3}, \ldots, x_{n}, t\right)=V\left(p\left(x_{1}, x_{2}, x_{3}, \ldots, x_{n}\right), t\right)$, where $p$ is a permutation function;

(VF-5) $V\left(x_{1}, x_{2}, x_{3}, \ldots, x_{n-1}, t+s\right) \geq V\left(x_{1}, x_{2}, x_{3}, \ldots, x_{n-1}, l, t\right) * V\left(l, l, l, \ldots, l, x_{n}, s\right)$;

(VF-6) $\lim _{t \rightarrow \infty} V\left(x_{1}, x_{2}, x_{3}, \ldots, x_{n}, t\right)=1$;

$(\mathrm{VF}-7) V\left(x_{1}, x_{2}, x_{3}, \ldots, x_{n}, \cdot\right):(0, \infty) \rightarrow[0,1]$ is continuous.

Example 2.1 Let $(X, A)$ be an $A$-metric space. Define the $t$-norm $a * b=a b$ or $a * b=$ $\min \{a, b\}$. For all $x_{1}, x_{2}, x_{3}, \ldots, x_{n} \in X, t>0$, denote

$$
V\left(x_{1}, x_{2}, x_{3}, \ldots, x_{n}, t\right)=\frac{t}{t+A\left(x_{1}, x_{2}, x_{3}, \ldots, x_{n}\right)} .
$$

Then $(X, V, *)$ is a $V$-fuzzy metric space.

Lemma 2.1 Let $(X, V, *)$ be a $V$-fuzzy metric space. Then $V\left(x_{1}, x_{2}, x_{3}, \ldots, x_{n}, t\right)$ is nondecreasing with respect to $t$. 
Proof Since $t>0$ and $t+s>t$ for $s>0$, by letting $l=x_{n}$ in condition (VF-5) of a $V$-fuzzy metric space, we get

$$
V\left(x_{1}, x_{2}, x_{3}, \ldots, x_{n-1}, x_{n}, t+s\right) \geq V\left(x_{1}, x_{2}, x_{3}, \ldots, x_{n-1}, x_{n}, t\right) * V\left(x_{n}, x_{n}, x_{n}, \ldots, x_{n}, x_{n}, s\right) .
$$

This implies that $V\left(x_{1}, x_{2}, x_{3}, \ldots, x_{n-1}, x_{n}, t+s\right) \geq V\left(x_{1}, x_{2}, x_{3}, \ldots, x_{n-1}, x_{n}, t\right)$.

So, $V\left(x_{1}, x_{2}, x_{3}, \ldots, x_{n-1}, x_{n}, t\right)$ is nondecreasing with respect to $t$.

Lemma 2.2 Let $(X, V, *)$ be a $V$-fuzzy metric space such that

$$
V\left(x_{1}, x_{2}, x_{3}, \ldots, x_{n}, k t\right) \geq V\left(x_{1}, x_{2}, x_{3}, \ldots, x_{n}, t\right)
$$

with $k \in(0,1)$. Then $x_{1}=x_{2}=x_{3}=\cdots=x_{n}$.

Proof By assumption

$$
V\left(x_{1}, x_{2}, x_{3}, \ldots, x_{n}, k t\right) \geq V\left(x_{1}, x_{2}, x_{3}, \ldots, x_{n}, t\right)
$$

for $t>0$. Since $k t<t$, by Lemma 2.1 we have

$$
V\left(x_{1}, x_{2}, x_{3}, \ldots, x_{n}, k t\right) \leq V\left(x_{1}, x_{2}, x_{3}, \ldots, x_{n}, t\right)
$$

From (1), (2), and the definition of a $V$-fuzzy metric space we get $x_{1}=x_{2}=x_{3}=\cdots=x_{n}$.

Definition 2.2 Let $(X, V, *)$ be a $V$-fuzzy metric space. A sequence $\left\{x_{r}\right\}$ is said to converge to a point $x \in X$ if $V\left(x_{r}, x_{r}, x_{r}, \ldots, x_{r}, x, t\right) \rightarrow 1$ as $r \rightarrow \infty$ for all $t>0$, that is, for each $\epsilon>0$, there exists $n \in N$ such that for all $r \geq N$, we have $V\left(x_{r}, x_{r}, x_{r}, \ldots, x_{r}, x, t\right)>1-\epsilon$, and we write $\lim _{r \rightarrow \infty} x_{r}=x$.

Definition 2.3 Let $(X, V, *)$ be a $V$-fuzzy metric space. A sequence $\left\{x_{r}\right\}$ is said to be a Cauchy sequence if $V\left(x_{r}, x_{r}, x_{r}, \ldots, x_{r}, x_{q}, t\right) \rightarrow 1$ as $r, q \rightarrow \infty$ for all $t>0$, that is, for each $\epsilon>0$, there exists $n_{0} \in N$ such that for all $r, q \geq n_{0}$, we have $V\left(x_{r}, x_{r}, x_{r}, \ldots, x_{r}, x_{q}, t\right)>1-\epsilon$.

Definition 2.4 The $V$-fuzzy metric space $(X, V, *)$ is said to be complete if every Cauchy sequence in $X$ is convergent.

Definition 2.5 The mappings $P: X \times X \rightarrow X$ and $Q: X \rightarrow X$ are said to be compatible on $V$-fuzzy metric spaces if

$$
\lim _{r \rightarrow \infty} V\left(Q P\left(x_{r}, y_{r}\right), Q P\left(x_{r}, y_{r}\right), \ldots, Q P\left(x_{r}, y_{r}\right), P\left(Q x_{r}, Q y_{r}\right), t\right)=1
$$

and

$$
\lim _{r \rightarrow \infty} V\left(Q P\left(y_{r}, x_{r}\right), Q P\left(y_{r}, x_{r}\right), \ldots, Q P\left(y_{r}, x_{r}\right), P\left(Q y_{r}, Q x_{r}\right), t\right)=1
$$

whenever $\left\{x_{r}\right\}$ and $\left\{y_{r}\right\}$ are sequences in $X$ such that $\lim _{r \rightarrow \infty} Q\left(x_{r}\right)=\lim _{r \rightarrow \infty} P\left(x_{r}, y_{r}\right)=x$ and $\lim _{r \rightarrow \infty} Q\left(y_{r}\right)=\lim _{r \rightarrow \infty} P\left(y_{r}, x_{r}\right)=y$ for all $x, y \in X$ and $t>0$. 


\section{Main results}

In this section, we explicitly prove fixed point theorems for coupled maps on partially ordered $V$-fuzzy metric spaces.

Theorem 3.1 Let $(X, V, *)$ be a complete $V$-fuzzy metric space, and $(X, \preceq)$ be a partially ordered set. Let $P: X \times X \rightarrow X$ and $Q: X \times X \rightarrow X$ be two mappings such that

(T1) $P(X \times X) \subseteq Q(X)$;

(T2) P has the mixed $Q$-monotone property;

(T3) there exists $k \in(0,1)$ such that

$$
\begin{aligned}
& V(P(x, y), P(x, y), \ldots, P(x, y), P(u, v), k t) \\
& \geq V(Q x, Q x, \ldots, Q x, Q u, t) \\
& \quad * V(Q x, Q x, \ldots, Q x, P(x, y), t) \\
& \quad * V(Q u, Q u, \ldots, Q u, P(u, v), t)
\end{aligned}
$$

for all $x, y, u, v \in X$ and $t>0$ for which $Q(x) \leq Q(u)$ and $Q(y) \geq Q(v)$ or $Q(x) \geq Q(u)$ and $Q(y) \leq Q(v)$;

(T4) $Q$ is continuous, and $P$ and $Q$ are compatible.

Also suppose that

(a) $P$ is continuous or

(b) X has the following properties:

(i) if $\left\{x_{r}\right\}$ is a nondecreasing sequence such that $x_{r} \rightarrow x$, then $x_{r} \leq x$ for all $r \in N$;

(ii) if $\left\{y_{r}\right\}$ is a nonincreasing sequence $y_{r} \rightarrow y$, then $y_{r} \geq y$ for all $r \in N$.

If there exist $x_{0}, y_{0} \in X$ such that $Q\left(x_{0}\right) \leq P\left(x_{0}, y_{0}\right)$ and $Q\left(y_{0}\right) \geq P\left(y_{0}, x_{0}\right)$, then $P$ and $Q$ have a coupled coincidence point in $X$.

Proof Let $\left(x_{0}, y_{0}\right)$ be a given point in $X \times X$ such that $Q\left(x_{0}\right) \leq P\left(x_{0}, y_{0}\right)$ and $Q\left(y_{0}\right) \geq$ $P\left(y_{0}, x_{0}\right)$. Using (T1), choose $x_{1}, y_{1}$ such that

$$
P\left(x_{0}, y_{0}\right)=Q\left(x_{1}\right) \text { and } P\left(y_{0}, x_{0}\right)=Q\left(y_{1}\right) \text {. }
$$

Construct two sequences $\left\{x_{r}\right\}$ and $\left\{y_{r}\right\}$ in $X$ such that $P\left(x_{r}, y_{r}\right)=Q\left(x_{r+1}\right)$ and

$$
P\left(y_{r}, x_{r}\right)=Q\left(y_{r+1}\right) \quad \text { for all } r \geq 0 .
$$

Now we shall prove that

$$
Q\left(x_{r}\right) \leq Q\left(x_{r+1}\right) \quad \text { and } \quad Q\left(y_{r}\right) \geq Q\left(y_{r+1}\right) .
$$

We use mathematical induction.

Step 1. Let $r=0$. Since $Q\left(x_{0}\right) \leq P\left(x_{0}, y_{0}\right)$ and $Q\left(y_{0}\right) \geq P\left(y_{0}, x_{0}\right)$, using condition (3), we have

$$
Q\left(x_{0}\right) \leq Q\left(x_{1}\right) \text { and } \quad Q\left(y_{0}\right) \geq Q\left(y_{1}\right)
$$

So inequalities (5) hold for $r=0$. 
Step 2. Now suppose that (5) hold for some fixed $s \geq 0$. So we get

$$
Q\left(x_{s}\right) \leq Q\left(x_{s+1}\right) \quad \text { and } \quad Q\left(y_{s}\right) \geq Q\left(y_{s+1}\right) .
$$

Step 3. Since $P$ has the mixed $Q$-monotone property, using (4), we have

$$
Q\left(x_{r+1}\right)=P\left(x_{r}, y_{r}\right) \leq P\left(x_{r+1}, y_{r}\right)
$$

and

$$
Q\left(y_{r+1}\right)=P\left(y_{r}, x_{r}\right) \geq P\left(y_{r+1}, x_{r}\right) .
$$

Also, $Q\left(x_{r+2}\right)=P\left(x_{r+1}, y_{r+1}\right) \geq P\left(x_{r+1}, y_{r}\right)$ and

$$
Q\left(y_{r+2}\right)=P\left(y_{r+1}, x_{r+1}\right) \leq P\left(y_{r+1}, x_{r}\right) .
$$

From (6) and (7) we get

$$
Q\left(x_{r}\right) \leq Q\left(x_{r+1}\right) \quad \text { and } \quad Q\left(y_{r}\right) \geq Q\left(y_{r+1}\right) .
$$

From (T3) and (4) we get

$$
\begin{aligned}
& V\left(P\left(x_{r-1}, y_{r-1}\right), P\left(x_{r-1}, y_{r-1}\right), \ldots, P\left(x_{r-1}, y_{r-1}\right), P\left(x_{r}, y_{r}\right), k t\right) \\
& \geq V\left(Q x_{r-1}, Q x_{r-1}, \ldots, Q x_{r-1}, Q x_{r}, t\right) \\
& \quad * V\left(Q x_{r-1}, Q x_{r-1}, \ldots, Q x_{r-1}, P\left(x_{r-1}, y_{r-1}\right), t\right) \\
& \quad * V\left(Q x_{r}, Q x_{r}, \ldots, Q x_{r}, P\left(x_{r}, y_{r}\right), t\right) \\
& V\left(Q x_{r}, Q x_{r}, \ldots, Q x_{r}, Q x_{r+1}, k t\right) \\
& \geq V\left(Q x_{r-1}, Q x_{r-1}, \ldots, Q x_{r-1}, Q x_{r}, t\right) \\
& \quad * V\left(Q x_{r}, Q x_{r}, \ldots, Q x_{r}, Q x_{r+1}, t\right) .
\end{aligned}
$$

Now, two cases arise.

Case 1. If $V\left(Q x_{r-1}, Q x_{r-1}, \ldots, Q x_{r-1}, Q x_{r}, t\right)<V\left(Q x_{r}, Q x_{r}, \ldots, Q x_{r}, Q x_{r+1}, t\right)$, then

$$
\begin{aligned}
V\left(Q x_{r}, Q x_{r}, \ldots, Q x_{r}, Q x_{r+1}, k t\right) \geq & V\left(Q x_{r-1}, Q x_{r-1}, \ldots, Q x_{r-1}, Q x_{r}, t\right) \\
\geq & V\left(Q x_{r-2}, Q x_{r-2}, \ldots, Q x_{r-2}, Q x_{r-1}, \frac{t}{k}\right) \\
\geq & V\left(Q x_{r-2}, Q x_{r-2}, \ldots, Q x_{r-2}, Q x_{r-1}, \frac{t}{k^{2}}\right) \\
& \cdots \\
\geq & V\left(Q x_{0}, Q x_{0}, \ldots, Q x_{0}, Q x_{1}, \frac{t}{k^{r-1}}\right) .
\end{aligned}
$$

Then by simple induction we have that, for all $t>0$ and $r=1,2, \ldots, \infty$,

$$
V\left(Q x_{r}, Q x_{r}, \ldots, Q x_{r}, Q x_{r+1}, t\right) \geq V\left(Q x_{0}, Q x_{0}, \ldots, Q x_{0}, Q x_{1}, \frac{t}{k^{r-1}}\right) .
$$


Thus, by condition (VF-5) of the definition of a $V$-fuzzy metric space, for any positive integer $p$ and real number $t>0$, we have

$$
\begin{aligned}
V\left(Q x_{r}, Q x_{r}, \ldots, Q x_{r}, Q x_{r+p}, t\right) \geq & V\left(Q x_{r}, Q x_{r}, \ldots, Q x_{r}, Q x_{r+1}, \frac{t}{p}\right) \\
& * V\left(Q x_{r+1}, Q x_{r+1}, \ldots, Q x_{r+1}, Q x_{r+2}, \frac{t}{p}\right) \\
& * \cdots p \text { times } \ldots \\
& * V\left(Q x_{r+p-1}, Q x_{r+p-1}, \ldots, Q x_{r+p-1}, Q x_{r+p}, \frac{t}{p}\right) \\
\geq & V\left(Q x_{0}, Q x_{0}, \ldots, Q x_{0}, Q x_{1}, \frac{t}{p k^{r-1}}\right) \\
& * \cdots p \text { times } \ldots \\
& * V\left(Q x_{0}, Q x_{0}, \ldots, Q x_{0}, Q x_{1}, \frac{t}{p k^{r+p-2}}\right) .
\end{aligned}
$$

Therefore, taking $r \rightarrow \infty$, by definition (VF-6) we get

$$
V\left(Q x_{r}, Q x_{r}, \ldots, Q x_{r}, Q x_{r+p}, t\right) \geq 1 * \cdots p \text { times } \cdots * 1,
$$

which implies that $\left\{Q x_{n}\right\}$ is a Cauchy sequence in $X$.

Case 2. If $V\left(Q x_{r-1}, Q x_{r-1}, \ldots, Q x_{r-1}, Q x_{r}, t\right)>V\left(Q x_{r}, Q x_{r}, \ldots, Q x_{r}, Q x_{r+1}, t\right)$, then

$$
V\left(Q x_{r}, Q x_{r}, \ldots, Q x_{r}, Q x_{r+1}, k t\right) \geq V\left(Q x_{r}, Q x_{r}, \ldots, Q x_{r}, Q x_{r+1}, t\right) .
$$

By Lemma 2.2 we get $Q x_{r}=Q x_{r+1}$.

Thus, there exists a positive integer $m$ such that $r \geq m$ implies $Q x_{r}=Q x_{m}, \forall r$, which shows that $\left\{Q x_{n}\right\}$ is a convergent sequence and so a Cauchy sequence in $X$.

Taking $x=y_{r}, y=x_{r}, u=y_{r-1}, v=x_{r-1}$ in (T3), we get

$$
\begin{aligned}
V( & \left.P\left(y_{r}, x_{r}\right), P\left(y_{r}, x_{r}\right), \ldots, P\left(y_{r}, x_{r}\right), P\left(y_{r-1}, x_{r-1}\right), k t\right) \\
\geq & V\left(Q y_{r}, Q y_{r}, \ldots, Q y_{r}, Q u, t\right) \\
& * V\left(Q y_{r}, Q y_{r}, \ldots, Q y_{r}, P\left(y_{r}, x_{r}\right), t\right) \\
& * V\left(Q y_{r-1}, Q y_{r-1}, \ldots, Q y_{r-1}, P\left(y_{r-1}, x_{r-1}\right), t\right) .
\end{aligned}
$$

So, from equation (4) we have

$$
\begin{aligned}
V\left(Q y_{r}, Q y_{r}, \ldots, Q y_{r}, Q y_{r+1}, k t\right) \geq & V\left(Q y_{r-1}, Q y_{r-1}, \ldots, Q y_{r-1}, Q y_{r}, t\right) \\
& * V\left(Q y_{r}, Q y_{r}, \ldots, Q y_{r}, Q y_{r+1}, t\right) .
\end{aligned}
$$

In the same way (discussed before), $\left\{Q y_{n}\right\}$ is a Cauchy sequence in $X$.

Since $X$ is a complete space, there exist $x, y \in X$ such that

$$
\lim _{r \rightarrow \infty} P\left(x_{r}, y_{r}\right)=\lim _{r \rightarrow \infty} Q\left(x_{r}\right)=x, \quad \lim _{r \rightarrow \infty} P\left(y_{r}, x_{r}\right)=\lim _{r \rightarrow \infty} Q\left(y_{r}\right)=y .
$$


By considering condition (T4) and $r \rightarrow \infty$ we have

$$
V\left(Q\left(P\left(x_{r}, y_{r}\right)\right), Q\left(P\left(x_{r}, y_{r}\right)\right), \ldots, Q\left(P\left(x_{r}, y_{r}\right)\right), P\left(Q\left(x_{r}\right), Q\left(y_{r}\right), t\right)\right) \rightarrow 1
$$

and

$$
V\left(Q\left(P\left(y_{r}, x_{r}\right)\right), Q\left(P\left(y_{r}, x_{r}\right)\right), \ldots, Q\left(P\left(y_{r}, x_{r}\right)\right), P\left(Q\left(y_{r}\right), Q\left(x_{r}\right), t\right)\right) \rightarrow 1
$$

as $r \rightarrow \infty$.

By conditions (T4) and (a), since $P$ and $Q$ are continuous, from (10) we have

$$
V(Q(x), Q(x), \ldots, Q(x), P(x, y), t)=1
$$

and

$$
V(Q(y), Q(y), \ldots, Q(y), P(y, x), t)=1 .
$$

This implies that $P(x, y)=Q(x)$ and $P(y, x)=Q(y)$, and thus, we have proved that $P$ and $Q$ have a coupled coincidence point in $X$.

Now, suppose that conditions (T4) and (b) hold. Since $Q$ is continuous and $P, Q$ are compatible mappings, we have

$$
\lim _{r \rightarrow \infty} P\left(Q\left(x_{r}\right), Q\left(y_{r}\right)\right)=\lim _{r \rightarrow \infty} Q\left(P\left(x_{r}, y_{r}\right)\right)=\lim _{r \rightarrow \infty} Q\left(Q x_{r}\right)=Q(x)
$$

and

$$
\lim _{r \rightarrow \infty} P\left(Q\left(y_{r}\right), Q\left(x_{r}\right)\right)=\lim _{r \rightarrow \infty} Q\left(P\left(y_{r}, x_{r}\right)\right)=\lim _{r \rightarrow \infty} Q\left(Q y_{r}\right)=Q(y) .
$$

By condition (VF-5) of a $V$-fuzzy metric space, as $r \rightarrow \infty$, we get

$$
\begin{aligned}
V(Q x, Q x, \ldots, Q x, P(x, y), t) \geq & V\left(Q x, Q x, \ldots, Q x, Q\left(Q x_{r+1}\right), t-k t\right) \\
& * V\left(Q\left(Q x_{r+1}\right), Q\left(Q x_{r+1}\right), \ldots, Q\left(Q x_{r+1}\right), P(x, y), k t\right) \\
= & V\left(Q x, Q x, \ldots, Q x, Q\left(P\left(x_{r}, y_{r}\right)\right), t-k t\right) \\
& * V\left(Q\left(P\left(x_{r}, y_{r}\right)\right), Q\left(P\left(x_{r}, y_{r}\right)\right), \ldots,\right. \\
& \left.Q\left(P\left(x_{r}, y_{r}\right)\right), P(x, y), k t\right) \\
\geq & V\left(Q\left(P\left(x_{r}, y_{r}\right)\right), Q\left(P\left(x_{r}, y_{r}\right)\right), \ldots,\right. \\
& \left.Q\left(P\left(x_{r}, y_{r}\right)\right), P(x, y), k t\right) .
\end{aligned}
$$

We get

$$
\begin{aligned}
V(Q x, Q x, \ldots, Q x, P(x, y), t) \geq & V\left(P\left(Q x_{r}, Q y_{r}\right), P\left(Q x_{r}, Q y_{r}\right), \ldots,\right. \\
& \left.P\left(Q x_{r}, Q y_{r}\right), P(x, y), k t\right) .
\end{aligned}
$$


Using condition (T3) and equations (11)-(12), we get

$$
\begin{aligned}
V(Q x, Q x, \ldots, Q x, P(x, y), t) \geq & V\left(Q\left(Q x_{r}\right), Q\left(Q x_{r}\right), \ldots, Q\left(Q x_{r}\right), Q x, t\right) \\
& * V\left(Q\left(Q x_{r}\right), Q\left(Q x_{r}\right), \ldots, P\left(Q x_{r}, Q y_{r}\right), t\right) \\
& * V(Q x, Q x, \ldots, Q x, P(x, y), t) \\
\geq & V(Q x, Q x, \ldots, Q x, P(x, y), t) .
\end{aligned}
$$

By Lemma 2.2 we have $P(x, y)=Q(x)$. Similarly, we get $P(y, x)=Q(y)$. Hence, we proved that $P$ and $Q$ have a coupled coincidence point in $X$.

Theorem 3.2 Assume that $X$ is a totally ordered set in addition to the hypotheses of Theorem 3.1. Then $P$ and $Q$ have a unique common coupled fixed point.

Proof Suppose that $(x, y)$ and $(l, m)$ are a coupled coincidence point of $P$ and $Q$, that is,

$$
P(x, y)=Q(x), P(y, x)=Q(y)
$$

and

$$
P(l, m)=Q(l), P(m, l)=Q(m) .
$$

Let us show that $Q(x)=Q(l), Q(y)=Q(m)$.

If $X$ is a totally ordered set, then for all $(x, y),(l, m) \in X \times X$, there exists $(\alpha, \beta) \in X \times X$ such that $(P(\alpha, \beta), P(\beta, \alpha))$ is comparable with $(P(x, y), P(y, x)),(P(l, m), P(m, l))$.

The sequences $\left\{Q\left(\alpha_{r}\right)\right\},\left\{Q\left(\beta_{r}\right)\right\}$ and their limits are defined similarly as in Theorem 3.1, so that

$$
P\left(\alpha_{r}, \beta_{r}\right)=Q\left(\alpha_{r+1}\right), \quad P\left(\beta_{r}, \alpha_{r}\right)=Q\left(\beta_{r+1}\right) .
$$

By condition (T3) we have

$$
\begin{aligned}
V\left(Q x, Q x, \ldots, Q x, Q \alpha_{r+1}, k t\right)= & V\left(Q x, Q x, \ldots, Q x, P\left(\alpha_{r}, \beta_{r}\right), t\right) \\
\geq & V\left(Q x, Q x, \ldots, Q x, Q \alpha_{r}, t\right) * V(Q x, Q x, \ldots, Q x, P(x, y), t) \\
& * V\left(Q \alpha_{r}, Q \alpha_{r}, \ldots, Q \alpha_{r}, P\left(\alpha_{r}, \beta_{r}\right), t\right) \\
\geq & V\left(Q x, Q x, \ldots, Q x, Q \alpha_{r}, t\right) \\
& * V\left(Q \alpha_{r}, Q \alpha_{r}, \ldots, Q \alpha_{r}, P\left(\alpha_{r}, \beta_{r}\right), t\right) .
\end{aligned}
$$

We obtained that

$$
Q(x)=Q(\alpha) \quad \text { as } r \rightarrow \infty .
$$

Again by (T3),

$$
\begin{aligned}
V\left(Q y, Q y, \ldots, Q y, Q \beta_{r+1}, k t\right) & =V\left(Q y, Q y, \ldots, Q y, P\left(\beta_{r}, \alpha_{r}\right), t\right) \\
& \geq V\left(Q y, Q y, \ldots, Q y, Q \beta_{r}, t\right)
\end{aligned}
$$




$$
\begin{aligned}
& * V(Q y, Q y, \ldots, Q y, P(y, x), t) \\
& * V\left(Q \beta_{r}, Q \beta_{r}, \ldots, Q \beta_{r}, P\left(\beta_{r}, \alpha_{r}\right), t\right) \\
\geq & V\left(Q y, Q y, \ldots, Q y, Q \alpha_{r}, t\right) \\
& * V\left(Q \alpha_{r}, Q \alpha_{r}, \ldots, Q \alpha_{r}, P\left(\beta_{r}, \alpha_{r}\right), t\right) .
\end{aligned}
$$

Letting $r \rightarrow \infty$, we have

$$
\lim _{r \rightarrow \infty} Q\left(\beta_{r}\right)=Q(y) .
$$

Following the above steps with the help of condition (T3), we obtain

$$
\lim _{r \rightarrow \infty} Q\left(\alpha_{r}\right)=Q(l), \quad \lim _{r \rightarrow \infty} Q\left(\beta_{r}\right)=Q(m) .
$$

From (13), (14), (15), and the definition of a $V$-fuzzy metric space we have

$$
\begin{aligned}
V(Q x, Q x, \ldots, Q x, Q l, t) \geq & V\left(Q x, Q x, \ldots, Q x, Q \alpha_{r+1}, \frac{t}{2}\right) \\
& * V\left(Q \alpha_{r+1}, Q \alpha_{r+1}, \ldots, Q \alpha_{r+1}, Q l, \frac{t}{2}\right) \rightarrow 1 \quad \text { as } r \rightarrow \infty .
\end{aligned}
$$

This implies that

$$
Q(x)=Q(l)
$$

Now, we can easily prove that

$$
Q(y)=Q(m)
$$

So, we have $P(x, y)=Q(x), P(y, x)=Q(y)$, and the compatibility of $P$ and $Q$ implies the $w$-compatibility of $P$ and $Q$ given by

$$
\begin{aligned}
& Q(Q(x))=Q(P(x, y))=P(Q(x), Q(y)), \\
& Q(Q(y))=Q(P(y, x))=P(Q(y), Q(x)) .
\end{aligned}
$$

This implies that $(Q(x), Q(y))$ is a coupled coincidence point.

Assuming that $l=Q(x), m=Q(y)$, by (16)-(17) we have

$$
Q(Q(x))=Q(x), \quad Q(Q(y))=Q(y) .
$$

From (18)-(19) we obtain

$$
Q(x)=Q(Q(x))=P(Q(x), Q(y))
$$

and

$$
Q(y)=Q(Q(y))=P(Q(y), Q(x)) .
$$

So, $(Q(x), Q(y))$ is a common coupled fixed point of $P$ and $Q$. 
We can easily prove the uniqueness of common coupled fixed point under the assumption that $\left(x^{*}, y^{*}\right)$ is another common coupled fixed point of $P$ and $Q$.

From (16)-(19) we can show that

$$
x^{*}=Q\left(x^{*}\right)=Q(Q(x))=Q(x)
$$

and

$$
y^{*}=Q\left(y^{*}\right)=Q(Q(y))=Q(y) .
$$

This implies that $P$ and $Q$ have a unique common coupled fixed point.

Here, we furnish an example to demonstrate the validity of the hypothesis of the above results.

Example 3.1 Let $(X, \leq)$ be a partially ordered set with $X=[0,1], a * b=\min \{a, b\}$. Let $P: X \times X \rightarrow X$ and $Q: X \rightarrow X$ be two mappings defined as

$$
P(x, y)=\left\{\begin{array}{ll}
\frac{x-y}{2} & \text { if } x \geq y, \\
0 & \text { if } x<y,
\end{array} \quad Q(x)=x .\right.
$$

This implies that $P$ satisfies the definition of the mixed Q-monotone property.

Let

$$
V\left(x_{1}, x_{2}, \ldots, x_{n}, t\right)=\frac{t}{t+A\left(x_{1}, x_{2}, \ldots, x_{n}\right)},
$$

where $A\left(x_{1}, x_{2}, \ldots, x_{n}\right)$ is the $A$-metric space defined as

$$
A\left(x_{1}, x_{2}, \ldots, x_{n}\right)=\left|x_{1}-x_{2}\right|+\left|x_{2}-x_{3}\right|+\cdots+\left|x_{n-1}-x_{n}\right|
$$

for all $x_{1}, x_{2}, \ldots, x_{n} \in X, t>0$.

Then $(X, V, *)$ is a complete $V$-fuzzy metric space.

We take $k=\frac{1}{2}$ and consider the sequences $\left\{x_{r}\right\},\left\{y_{r}\right\}$ in $X$ defined by $x_{r}=\frac{1}{2 r}, y_{r}=\frac{1}{3 r}$.

Since

$$
\begin{aligned}
& \lim _{r \rightarrow \infty} P\left(x_{r}, y_{r}\right)=\lim _{r \rightarrow \infty} Q\left(x_{r}\right)=0=w \quad \text { (say), } \\
& \lim _{r \rightarrow \infty} P\left(y_{r}, x_{r}\right)=\lim _{r \rightarrow \infty} Q\left(y_{r}\right)=0=w^{\prime} \quad \text { (say). }
\end{aligned}
$$

Also, $P: X \times X \rightarrow X$ and $Q: X \rightarrow X$ are compatible mappings in $X$. From Theorem 3.1 we have that $Q(x) \leq Q(u)$ and $Q(y) \geq Q(v)$. This implies $x \leq u, y \geq v$. If we consider $x \geq y$, $u \geq v$, then we have

$$
\begin{gathered}
V\left(P(x, y), P(x, y), \ldots, P(x, y), P(u, v) \frac{t}{2}\right) \\
=\frac{\frac{t}{2}}{\frac{t}{2}+2\left|\frac{(x-y)-(u-v)}{2}\right|} \geq \frac{t}{t+|u+v|}
\end{gathered}
$$




$$
\begin{aligned}
= & V(Q(u), Q(u), \ldots, Q(u), P(u, v), t) \\
\geq & V(Q(x), Q(x), \ldots, Q(x), Q(u), t) \\
& * V(Q(x), Q(x), \ldots, Q(x), P(x, y), t) \\
& * V(Q(u), Q(u), \ldots, Q(u), P(u, v), t) .
\end{aligned}
$$

If we consider $x<y, u \geq v$, then we have

$$
\begin{aligned}
V( & \left(x(x, y), P(x, y), \ldots, P(x, y), P(u, v) \frac{t}{2}\right) \\
= & \frac{\frac{t}{2}}{\frac{t}{2}+2\left|\frac{(u-v)}{2}\right|} \geq \frac{t}{t+2|u-x|} \\
\geq & V(Q(x), Q(x), \ldots, Q(x), Q(u), t) \\
\geq & V(Q(x), Q(x), \ldots, Q(x), Q(u), t) \\
& * V(Q(x), Q(x), \ldots, Q(x), P(x, y), t) \\
& * V(Q(u), Q(u), \ldots, Q(u), P(u, v), t) .
\end{aligned}
$$

If we consider $x<y, u<v$, then we get directly condition (T3) of Theorem 3.1.

Therefore, all hypotheses of Theorem 3.1 hold. So we conclude that $\left(w, w^{\prime}\right)$ is a common coupled fixed point of $P$ and $Q$.

Theorem 3.3 Let $(X, V, *)$ be a complete $V$-fuzzy metric space, and $(X, \leq)$ be a partially ordered set. Let $P: X \times X \rightarrow X$ be a mapping such that $P$ has the mixed monotone property and there exists $k \in(0,1)$ such that

$$
\begin{aligned}
& V(P(x, y), P(x, y), \ldots, P(x, y), P(u, v), k t) \\
& \quad \geq V(x, x, \ldots, x, u, t) * V(x, x, \ldots, x, P(x, y), t) \\
& \quad * V(u, u, \ldots, u, P(u, v), t)
\end{aligned}
$$

for all $x, y, u, v \in X, t>0$ such that $x \leq u$ and $y \geq v$.

Also suppose that

(a) $P$ is continuous or

(b) $X$ has the following properties:

(i) if $\left\{x_{n}\right\}$ is a nondecreasing sequence $x_{r} \rightarrow x$, then $x_{r} \leq x$ for all $r \in N$,

(ii) if $\left\{y_{n}\right\}$ is a nondecreasing sequence $y_{r} \rightarrow y$, then $y_{r} \geq x$ for all $r \in N$.

If there exist $x_{0}, y_{0} \in X$ such that $x_{0} \leq P\left(x_{0}, y_{0}\right)$ and $y_{0} \geq P\left(y_{0}, x_{0}\right)$, then $P$ has a coupled fixed point in $X$.

Proof By assuming $Q=I$ (the identity mapping) in Theorem 3.1 we get the result.

\section{Symmetric $V$-fuzzy metric space}

Definition 4.1 A $V$-fuzzy metric space $(X, V, *)$ is said to be symmetric if

$$
V(x, x, x, \ldots, x, y, t)=V(x, y, y, \ldots, y, y, t)
$$

for all $x, y \in X, t>0$. 
Remark 4.1 If $(X, M, *)$ is a fuzzy metric space, then $(X, V, *)$ is a $V$-fuzzy metric space, where

$$
V\left(x_{1}, x_{2}, \ldots, x_{n}, t\right)=\min \left\{M\left(x_{1}, x_{2}, t\right), M\left(x_{2}, x_{3}, t\right), \ldots, M\left(x_{n-1}, x_{n}, t\right), M\left(x_{n}, x_{1}, t\right)\right\}
$$

Now we have

$$
\begin{aligned}
& V\left(x_{1}, x_{2}, \ldots, x_{n-1}, l, t\right) * V\left(l, l, \ldots, l, x_{n}, s\right) \\
&= M\left(x_{n}, l, s\right) * \min \left\{M\left(x_{1}, x_{2}, t\right), M\left(x_{2}, x_{3}, t\right), \ldots, M\left(x_{n-1}, l, t\right), M\left(l, x_{1}, t\right)\right\} \\
& \leq \min \left\{M\left(x_{n}, l, s\right) * M\left(x_{1}, x_{2}, t\right), M\left(x_{n}, l, s\right) * M\left(x_{2}, x_{3}, t\right), \ldots,\right. \\
&\left.M\left(x_{n}, l, s\right) * M\left(x_{n-1}, l, t\right), M\left(x_{n}, l, s\right) * M\left(l, x_{1}, t\right)\right\} \\
& \leq \min \left\{M\left(x_{1}, x_{2}, t\right), M\left(x_{2}, x_{2}, t\right), \ldots, M\left(x_{n}, x_{n-1}, t\right), M\left(x_{n}, x_{1}, t+s\right)\right\} \\
& \leq \min \left\{M\left(x_{1}, x_{2}, t+s\right), M\left(x_{2}, x_{2}, t+s\right), \ldots, M\left(x_{n}, x_{n-1}, t+s\right), M\left(x_{n}, x_{1}, t+s\right)\right\} \\
&= V\left(x_{1}, x_{2}, \ldots, x_{n-1}, x_{n}, t+s\right),
\end{aligned}
$$

which implies

$$
V\left(x_{1}, x_{2}, \ldots, x_{n-1}, l, t\right) * V\left(l, l, \ldots, l, x_{n}, s\right) \leq V\left(x_{1}, x_{2}, \ldots, x_{n-1}, x_{n}, t+s\right) .
$$

So, all conditions of a $V$-fuzzy metric space are satisfied.

Remark 4.2 If $(X, V, *)$ is a symmetric $V$-fuzzy metric space and $V(x, x, \ldots, y, t)=$ $M(x, y, t)$ (using Remark 4.1), then $(X, M, *)$ is a fuzzy metric space.

By this remark we get the following desired result.

Theorem 4.1 Let $(X, M, *)$ be a complete fuzzy metric space, and $(X, \preceq)$ be a partially ordered set. Let $P: X \times X \rightarrow X$ and $Q: X \rightarrow X$ be two mappings such that

(T1) $P(X \times X) \subseteq Q(X)$;

(T2) $P$ has the mixed $Q$-monotone property;

(T3) there exists $k \in(0,1)$ such that

$$
M(P(x, y), P(u, v), k t) \geq M(Q x, Q u, t) * M(Q x, P(x, y), t) * M(Q u, P(u, v), t)
$$

for all $x, y, u, v \in X, t>0$ such that $Q(x) \leq Q(u)$ and $Q(y) \geq Q(v)$ or $Q(x) \geq Q(u)$ and $Q(y) \leq Q(v)$;

(T4) $Q$ is continuous, and $P$ and $Q$ are compatible.

$$
\text { Also suppose that }
$$

(a) $P$ is continuous or

(b) $X$ has the following properties:

(i) if $\left\{x_{r}\right\}$ is a nondecreasing sequence $x_{r} \rightarrow x$, then $x_{r} \leq x$ for all $r \in N$;

(ii) if $\left\{y_{r}\right\}$ is a nondecreasing sequence $y_{r} \rightarrow y$, then $y_{r} \leq x$ for all $r \in N$.

If there exist $x_{0}, y_{0} \in X$ such that $Q\left(x_{0}\right) \leq P\left(x_{0}, y_{0}\right)$ and $Q\left(y_{0}\right) \geq P\left(y_{0}, x_{0}\right)$, then $P$ and $Q$ have a coupled coincidence point in $X$. 


\section{Competing interests}

The authors declare that they have no competing interests.

\section{Authors' contributions}

Each author contributed equally in the development of this manuscript. Both authors read and approved the final version of this manuscript.

Received: 10 December 2015 Accepted: 29 March 2016 Published online: 12 April 2016

\section{References}

1. Mustafa, Z, Sims, B: A new approach to generalized metric spaces. J. Nonlinear Convex Anal. 7(2), 289-297 (2006)

2. Chen, J, Huang, X: Coupled fixed point theorems for compatible mappings in partially ordered $G$-metric spaces. J. Nonlinear Sci. Appl. 8(2), 130-141 (2015)

3. Chen, J, Huang, X: Quadruple fixed point theorems under $(\varphi, \psi)$-contractive conditions in partially ordered G-metric spaces with mixed $g$-monotone property. J. Nonlinear Sci. Appl. 8(4), 285-300 (2015)

4. Tu, Q, Zhu, C, Wu, Z: Common fixed point theorems under strict contractive conditions in Menger probabilistic G-metric spaces. J. Nonlinear Sci. Appl. 8(6), 1176-1189 (2015)

5. Sedghi, S, Shobe, N, Aliouche, A: A generalization of fixed point theorems in S-metric spaces. Mat. Vesn. 64(3), 258-266 (2012)

6. Abbas, M, Ali, B, Suleiman, Yl: Generalized coupled common fixed point results in partially ordered A-metric spaces. Fixed Point Theory Appl. 2015, 64 (2015). doi:10.1186/s13663-015-0309-2

7. Zadeh, LA: Fuzzy sets. Inf. Control 8, 338-353 (1965)

8. George, A, Veeramani, P: On some results in fuzzy metric spaces. Fuzzy Sets Syst. 64, 395-399 (1994)

9. Kramosil, I, Michalek, J: Fuzzy metric and statistical metric spaces. Kybernetika 11, 336-344 (1975)

10. Schweizer, B, Sklar, A: Statistical metric spaces. Pac. J. Math. 10, 314-334 (1960)

11. Mishra, SN, Sharma, N, Singh, SL: Common fixed points of maps on fuzzy metric spaces. Int. J. Math. Sci. 17, 253-258 (1994)

12. Sun, G, Yang, K: Generalized fuzzy metric spaces with properties. Res. J. Appl. Sci. 2, 673-678 (2010)

13. Bhaskar, TG, Lakshmikantham, V: Fixed point theorems in partially ordered metric spaces and applications. Nonlinear Anal. TMA 65, 1379-1393 (2006)

14. Lakshmikantham, V, Ciric, LB: Coupled fixed point theorems for nonlinear contractions in partially ordered metric space. Nonlinear Anal. TMA 70, 4341-4349 (2009)

15. Choudhurya, BS, Kundu, A: A coupled coincidence point result in partially ordered metric spaces for compatible mappings. Nonlinear Anal. 73, 2524-2531 (2010)

16. Abbas, M, Ali, M, Radenovic, S: Common coupled fixed point theorems in cone metric spaces for $w$-compatible mappings. Appl. Math. Comput. 217, 195-202 (2010)

17. Ciric, LB, Abbas, M, Damjanovic, B, Saadati, R: Common fuzzy fixed point theorems in ordered metric spaces. Math. Comput. Model. 53, 1737-1741 (2011)

18. Gupta, V, Kanwar, A: Fixed point theorem in fuzzy metric spaces satisfying E.A property. Indian J. Sci. Technol. 5, 3767-3769 (2012)

19. Gupta, V, Mani, N: Existence and uniqueness of fixed point in fuzzy metric spaces and its applications. In: Proceedings of the Second International Conference on Soft Computing for Problem Solving. Advances in Intelligent Systems and Computing, vol. 236, pp. 217-224. Springer, Berlin (2014)

20. Gupta, V, Saini, RK, Mani, N, Tripathi, AK: Fixed point theorems using control function in fuzzy metric spaces. Cogent Math. 2, Article ID 1053173 (2015)

21. Hu, X, Luo, Q: Coupled coincidence point theorems for contractions in generalized fuzzy metric spaces. Fixed Point Theory Appl. 2012, 196 (2012). doi:10.1186/1687-1812-2012-196

\section{Submit your manuscript to a SpringerOpen ${ }^{\circ}$ journal and benefit from:}

- Convenient online submission

Rigorous peer review

- Immediate publication on acceptance

- Open access: articles freely available online

- High visibility within the field

- Retaining the copyright to your article 